\title{
Papillomavirus DNA in sperm from infertile patients
}

\author{
William Gennari, Rita Bianchi', Anna Maria Teresa Sabbatini, Rita Magnani, Antonella \\ Grottola, Sandra Mattioli, Monica Pecorari, Fabio Rumpianesi \\ S.C. Microbiologia e Virologia, Azienda Ospedaliero-Universitaria \\ I UO Ginecologia e Ostetricia Ospedale di Carpi (Mo) Azienda USL
}

Key words: Papillomavirus DNA, sperm, infertile patients

Presenza di Papillomavirus DNA in spermatozoi di pazienti infertili e possibile significato clinico

\section{SUMMARY}

The Human Papillomaviruses (HPV) are causative agents of sexually transmitted disease that affect both men and women $(6,7)$. The genus includes more than 150 types divided in according to different tropism for skin surfaces and paved mucosal epithelia. Although HPV infection has a very high incidence in both sexes, HPV infection in men is often neglected because of its transitory nature and its lack of clinical relevance. The HPV infection in males was found to be borne by the anal region, perineum, scrotum, urethra and glans. The persistence of the virus in these sites of infection has been linked both to male infertility and to the development of neoplasia in genital areas and not. In addition, several studies have documented the presence of HPV in the semen but with conflicting results regarding the location of the virus in the various components of semen $(5,9,10)$.

The objective of this study was to highlight the presence of HPV DNA in the sperm of patients waiting for a Medically Assisted Procreation and to evaluate if there is a correlation between the semen parameters (motility, concentration and morphology of spermatozoa) and HPV infection.

\section{INTRODUZIONE}

I Papillomavirus umani (HPV) sono agenti causali di patologia virale prevalentemente a trasmissione sessuale che colpisce sia uomini che donne $(6,7)$. Il genere comprende più di 150 tipi suddivisibili a seconda del differente tropismo per la pelle e le superfici cutanee e per gli epiteli pavimentati delle mucose. Nonostante l'infezione da HPV abbia un'incidenza molto elevata in entrambi i sessi, spesso l'infezione da HPV nel sesso maschile è trascurata perché ritenuta transitoria $\mathrm{e}$ di scarsa rilevanza clinica. Nei maschi l'infezione da HPV è stata riscontrata a carico delle regioni anale, perineale, scrotale, uretrale, del glande e dell'asta. La persistenza del virus in questi siti di infezione è stata messa in relazione sia all'infertilità maschile che allo sviluppo di neoplasie a carico di aree genitali e non genitali. Inoltre, diversi lavori hanno documentato la presenza del virus HPV nel liquido seminale ma con risultati contrastanti per quanto concerne la localizzazione del virus nell'ambito delle diverse componenti del seme $(4,8-10)$.

L'obiettivo del presente studio è stato quello di evidenziare la presenza di HPV DNA negli spermatozoi di pazienti in attesa di un protocollo di Procreazione Medicalmente Assistita (PMA) e di valutare i parametri seminali (motilità, concentrazione per $\mathrm{ml}$ e morfologia degli spermatozoi) in relazione all'infezione ed al genotipo di HPV.

\section{MATERIALI E METODI}

Questo studio è stato approvato dal Comitato Etico dell'Azienda Ospedaliero-Universitaria di Modena e condotto in completo anonimato secondo i principi definiti dalla Conferenza di Helsinki sui diritti del paziente. Al momento dell'arruolamento ai pazienti è stato richiesto un consenso informato scritto.

\section{Pazienti}

Sono stati arruolati 51 pazienti maschi infertili tra i partners delle coppie afferenti all'ambulatorio della sterilità di coppia dell'Ospedale di Carpi (MO) che si dovevano sottoporre a spermiogramma per un protocollo di PMA di $1^{\circ}$ livello (inseminazione intrauterina), di età compresa tra $25 \mathrm{e}$ 50 anni e sierologicamente negativi sia per le malattie virali (HIV, HCV, HBV) sia per sifilide. Nessuno dei pazienti presentava malattie sessualmente trasmissibili evidenti come condilomi da HPV, herpes genitale o infezioni da Chlamydia trachomatis e da Neisseria gonorrhoeae.

Sono stati considerati come infertili quei soggetti che avevano avuto rapporti sessuali non protetti

\section{Corresponding author: Monica Pecorari}

Struttura Complessa di Microbiologia e Virologia, Azienda Integrata Ospedaliero-Universitaria, Policlinico Via del Pozzo, 7I - 4II 24 Modena - Tel.: 059-4223758 - Fax: 059-4223625;

E-mail: pecorari.monica@policlinico.mo.it 
per la ricerca di prole da almeno 18 mesi con alterazione dei parametri seminali e con partner normale per l'esclusione di anomalie tubariche, uterine, cervicali, ovariche ed endocrine. Per ogni paziente è stata raccolta una anamnesi che includeva pregressi interventi chirurgici, fumo e abitudini sessuali. Criteri di esclusione dallo studio sono stati i seguenti: pregresso criptorchidismo, pregressi traumi testicolari e orchiti post-parotitiche. Sono stati inoltre esclusi i soggetti con varicocele e i pazienti con spermiocoltura positiva per miceti o batteri. Tutti i soggetti hanno eseguito la raccolta del liquido seminale per la valutazione standard del liquido seminale.

\section{Processazione dei campioni seminali}

I campioni ottenuti per masturbazione dopo 2-7 giorni massimo di astinenza sessuale sono stati valutati, previo processo di liquefazione, in maniera quali-quantitativa (volume, $\mathrm{pH}$, aspetto, viscosità, concentrazione, morfologia e motilità nemaspermica) secondo i criteri delle linee guida del WHO "Laboratory Manual for the Examination of Human Semen and SpermCervical Mucus Interaction, 1999 4th edition" (11) analizzando sia i parametri macroscopici che quelli microscopici (Tabella 1). Parte del campione è stato poi utilizzato per effettuare l'esame che riproduce in vitro le modificazioni (capacitazione) a cui va incontro lo spermatozoo mentre attraversa le vie genitali femminili. La restante parte è stata congelata $-20^{\circ} \mathrm{C}$ in provette sterili da $2 \mathrm{ml}$ fino al momento dell'estrazione degli acidi nucleici.

\section{Estrazione DNA dagli spermatozoi}

Il liquido seminale è stato scongelato in bagno termostatato a $37^{\circ} \mathrm{C}$ e $1 \mathrm{ml}$ di campione risospeso è stato trasferito in provetta e centrifugato a 2.500 rpm per 10 minuti e la parte cellulare, recuperata e lavata in soluzione salina tamponata (PBS 1X), è stata sottoposta ad estrazione del DNA mediante metodica home-brew (12).

\section{Amplificazione dell'acido nucleico}

L'amplificazione ha interessato regioni genomiche di HPV diverse: la regione L1, altamente conservata, la cui amplificazione permette di identificare i principali genotipi HPV ad alto rischio (HPV 16, 18, 31, 33, 35, 39, 45, 51, 52, 54, 56, 58) ed a basso rischio e (HPV 6, 11) e le regioni genomiche E6/E7 la cui amplificazione permette di identificare i genotipi ad alto rischio (HPV 16, 18, $31,33,52,58)$.

L'amplificazione del DNA delle regioni geniche L1 ed E6/E7 è stata condotta in nested PCR. Per l'amplificazione è stata utilizzata una quantità di
1,5 ug di DNA estratto per ciascun campione in un volume finale di 50 ul contenenti 20 pmoli di ciascun primer e 25 ul di DreamTaq Green PCR Master Mix 2X (Fermentas International Inc., Canada). Successivamente 1,5 ul del prodotto di amplificazione della PCR esterna è stato sottoposto a nested PCR.

L'amplificazione della regione genomica L1 è stata eseguita utilizzando i primers MY09 e MY11(1). I campioni sono stati sottoposti a 40 cicli di amplificazione $\left(95^{\circ} \mathrm{C}-1\right.$ ', $55^{\circ} \mathrm{C}-1$ ', $72^{\circ} \mathrm{C}$ $\left.1^{\prime}\right)$ con una denaturazione iniziale a $95^{\circ} \mathrm{C}-2^{\prime}$ ' ed una estensione finale a $55^{\circ} \mathrm{C}-2^{\prime} \mathrm{e} 72^{\circ} \mathrm{C}-5^{\prime}$. La PCR interna è stata effettuata utilizzando i primers GP5+ e GP6+ (3) e sottoponendo i campioni a 35 cicli di amplificazione $\left(95^{\circ} \mathrm{C}-30 ", 40^{\circ} \mathrm{C}-45^{\prime}\right.$, $\left.72^{\circ} \mathrm{C}-30^{\prime \prime}\right)$ con una denaturazione iniziale a $95^{\circ} \mathrm{C}$ $8^{\prime}$ ed una estensione finale a $72^{\circ} \mathrm{C}-3$ '.

La regione genomica E6/E7 è stata amplificata utilizzando i primers LCRS e E7AS (5). I campioni sono stati sottoposti, previa denaturazione a $95^{\circ} \mathrm{C}-5$ ', a 10 cicli iniziali di amplificazione (5 cicli a $94^{\circ} \mathrm{C}-1^{\prime}, 45^{\circ} \mathrm{C}-1,, 72^{\circ} \mathrm{C}-2$ ' seguiti da 5 cicli a $94^{\circ} \mathrm{C}-1{ }^{\prime}, 48^{\circ} \mathrm{C}-1$ '), $72^{\circ} \mathrm{C}-1$ ', seguiti da altri 30 cicli $\left(94^{\circ} \mathrm{C}-1^{\prime}, 55^{\circ} \mathrm{C}-1^{\prime}, 72^{\circ} \mathrm{C}-1^{\prime}\right)$. L'estensione finale è stata effettuata a $55^{\circ} \mathrm{C}-2^{\prime}$ e $72^{\circ} \mathrm{C}-5^{\prime}$. La $\mathrm{PCR}$ interna è stata condotta utilizzando i primers pU-1M e pU-2R (5), con una denaturazione iniziale a $95^{\circ} \mathrm{C}-5^{\prime}$, seguita da 30 cicli di amplificazione $\left(94^{\circ} \mathrm{C}-1^{\prime}, 55^{\circ} \mathrm{C}-2^{\prime}, 72^{\circ} \mathrm{C}-2^{\prime}\right)$ e con una estensione finale a $55^{\circ} \mathrm{C}-2$ ' $\mathrm{e} 72^{\circ} \mathrm{C}-5^{\prime}$.

Per verificare l'eventuale presenza di inibitori della PCR, sui campioni è stata effettuata l'amplificazione di un frammento del gene della $\beta$-globina.

I prodotti di PCR in quantità di $10 \mu \mathrm{l}$ per ciascun campione sono stati sottoposti a elettroforesi su gel di agarosio al 3\% contenente bromuro d'etidio.

\section{Genotipizzazione di HPV}

I campioni risultati positivi all'amplificazione della regione L1 e quelli risultati positivi per la regione E6/E7 sono stati sottoposti a genotipizzazione allo scopo di definire il tipo di HPV. Per la genotipizzazione dei campioni risultati positivi all'amplificazione della regione L1, si è proceduto con un due kits commerciali basati sulla tecnologia LiPA a ibridazione inversa (Linear Array ${ }^{\circledR}$ HPV Genotyping Test, Roche Diagnostics SpA, Monza, Milano, Italia e Innogenetics Srl, Gent, Belgium) uno a conferma dell'altro, mentre i campioni risultati positivi per la regione E6/E7 sono stati genotipizzati tramite l'utilizzo di enzimi di restrizione specifici secondo una metodica RFLP home-brew (5).

\section{Statistica}

I risultati descritti nel presente studio sono stati 
elaborati mediante SPSS (Statistical Package for Social Science, SSPSS Inc., Chicago, Illinois) Versione 13.00 e il test $\chi^{2}$ è stato utilizzato per l'analisi statistica. Valori uguali od inferiori a $\mathrm{p}<0.05$ sono stati considerati significativi.

\section{RISULTATI}

Nessuno dei 51 pazienti arruolati per lo studio presentava malattie sessualmente trasmissibili evidenti come condilomi da HPV, herpes genitale o infezioni da Chlamydia trachomatis e da Neisseria gonorrhoeae. Lo stato seminale di positività per HPV non era associabile con alcuna caratteristica o storia clinica pregressa di infezione da HPV.

La valutazione dei parametri seminali ottenuta mediante spermiogramma mostrava l'assenza in tutti i pazienti di un quadro di normozoospermia. In particolare 22 pazienti su 51 (43\%) evidenziavano un quadro di oligozoospermia (concentrazione di spermatozoi/ml inferiore a $\left.20 \times 10^{6}\right), 51$ pazienti su $51(100 \%)$ presentavano un quadro di motilità nemaspermica rettilinea progressiva inferiore ai valori di riferimento, 41 pazienti su 51 $(80 \%)$ presentavano un quadro di motilità totale (motilità rettilinea progressiva + motilità ondulatoria) inferiore al $50 \%$ e infine 50 su 51 pazienti (98\%) mostravano all'analisi seminale una percentuale di spermatozoi con morfologia anomala superiore al limite di norma (maggiore al $70 \%$ ) (Tabella 2).

Per la ricerca di HPV è stato possibile ottenere DNA totale dagli spermatozoi in quantità sufficiente per l'amplificazione di 49 campioni seminali su 51 raccolti (96\%). L'assenza di inibitori della PCR è stata accertata su tutti i 49 campioni I campioni positivi per HPV sono risultati in totale 9 su $49(18 \%)$ di cui 7 positivi all'amplificazione della regione L1 e 2 positivi all'amplificazione delle regioni E6/E7.

La genotipizzazione (Tabella 3 ) è stata eseguita sui 7 campioni positivi per la regione L1 mediante il kit Linear Array ${ }^{\circledast}$ HPV Genotyping e kit LiPA Innogenetics. Con il test Linear Array ${ }^{\circledR}$ HPV Genotyping è stato possibile identificare 4/7 genotipi di HPV; in particolare sono stati riscontrati i genotipi HPV 16 nel campione 1, HPV 73 nel campione 4, HPV 31 nel campione 5, mentre nel campione 6 erano presenti in associazione $i$ genotipi HPV 31 e HPV 81. I campioni 2, 3 e 7, risultati non tipizzabili con tale metodica, sono stati sottoposti a tipizzazione con il kit LiPA Innogenetics. Con questa metodica è stato possibile evidenziare i genotipi HPV 52 in associazione a HPV 44 nel campione 2, HPV 16 nel campione 3 , mentre il campione 7 è risultato non tipizzabile anche con questo metodo. I campioni 8 e 9, risultati positivi all'amplificazione della regione E6/E7, sono stati identificati tramite RFLP sul frammento di PCR amplificato nella regione E6/E7, come HPV 31.

Nella Tabella 4 sono riportati i dati relativi alla presenza di HPV in associazione con i parametri seminali: HPV è stato ritrovato in 6 campioni su 20 oligozoospermici $(30 \%)$ e in 3 su $29(10 \%)$ campioni con concentrazione nemaspermica normale. Per quanto riguarda la motilità rettilinea, 9 campioni su 49 patologici (18\%) sono risultati HPV positivi. Considerando la motilità totale (rettilinea + ondulatoria) 6 campioni positivi per HPV su $39(15 \%)$ presentavano una motilità alterata mentre 3 campioni positivi su $10(30 \%)$ avevano motilità totale nella norma. L'unico campione che presentava morfologia tipica entro i limiti di norma era negativo per la ricerca di HPV mentre 9 campioni con morfologia atipica su 48 (19\%) sono risultati positivi.

\section{DISCUSSIONE}

Si definisce infertilità quella condizione per cui dopo un anno di rapporti sessuali non protetti una coppia non riesce ad avere un figlio. L'infertilità è una condizione che riguarda dal $15 \%$ al $20 \%$ delle coppie in età fertile. Sebbene la causa di infertilità di una coppia dipenda per un terzo esclusivamente dall'uomo, sembra a tutt'oggi in aumento sia per lo stile di vita sia per fattori ambientali, come l'inquinamento, che potrebbero giocare a questo proposito un ruolo rilevante. Diverse sono le cause documentate che portano all'infertilità maschile tra cui le infezioni provocate da malattie veneree (gonorrea) o da altre malattie sessualmente trasmissibili come l'infezione da Chlamydia trachomatis. Un ruolo nell'infertilità maschile può essere attribuito ad HPV; numerosi studi riportati in letteratura hanno dimostrato la presenza di HPV nel liquido seminale di uomini infertili, associandola ad alterazioni del $\mathrm{pH}$ del plasma seminale (10) e ad alterazioni nella motilità degli spermatozoi (4).

In accordo con tali dati, il presente studio, ha evidenziato la presenza di HPV DNA negli spermatozoi nel $18 \%$ dei pazienti infertili considerati. Questa percentuale di positività per HPV nei campioni presi in esame, considerando comunque la bassa casistica, risulta essere più alta rispetto ai dati di positività percentuale riportati in letteratura su una popolazione analoga di pazienti (2).

All'amplificazione in PCR della regione L1 sono risultati positivi 7 campioni mentre 2 campioni sono risultati positivi solamente all'amplificazione della regione E6/E7. Questi dati indicano che la scelta di amplificare la regione E6/E7, oltre la regione L1, è risultata ottimale perché ha permes- 
Tabella I. Parametri seminali microscopici dei campioni raccolti

\begin{tabular}{|c|c|c|c|c|c|c|c|}
\hline $\mathrm{Pz} \mathrm{N}^{\circ}$ & $\begin{array}{c}\text { Numero } \\
10^{6} / \mathrm{ml}\end{array}$ & $\begin{array}{l}\% \text { Motilità } \\
\text { rettilinea }(\mathrm{A})\end{array}$ & $\begin{array}{c}\text { \% Motilità } \\
\text { ondulatoria (B) }\end{array}$ & $\%(A+B)$ & $\begin{array}{c}\text { \% Motilità } \\
\text { in loco }\end{array}$ & $\begin{array}{c}\text { \% Spermatozoi } \\
\text { immobili }\end{array}$ & $\begin{array}{c}\text { \% Morfologia } \\
\text { atipica }\end{array}$ \\
\hline $\mathrm{I}$ & 7 & 0 & 40 & 40 & 30 & 30 & 74 \\
\hline 2 & 38 & 0 & 33 & 33 & 33 & 34 & 70 \\
\hline 3 & 27 & 0 & 30 & 30 & 25 & 45 & 72 \\
\hline 4 & 30 & 0 & 33 & 33 & 23 & 44 & 70 \\
\hline 5 & 25 & 8 & 32 & 40 & 25 & 35 & 73 \\
\hline 6 & 3 & 0 & 40 & 40 & 15 & 45 & 70 \\
\hline 7 & 33 & 0 & 30 & 30 & 40 & 30 & 75 \\
\hline 8 & 25 & 15 & 34 & 49 & 8 & 43 & 72 \\
\hline 9 & 3 & 3 & 32 & 35 & 35 & 30 & 75 \\
\hline 10 & 26 & 0 & 8 & 8 & 12 & 80 & 78 \\
\hline 11 & 24 & 0 & 48 & 48 & 23 & 29 & 72 \\
\hline 12 & 14 & 14 & 36 & 50 & 24 & 26 & 78 \\
\hline 13 & 6 & 0 & 20 & 20 & 20 & 60 & 70 \\
\hline 14 & 12 & 5 & 38 & 43 & 13 & 44 & 78 \\
\hline 15 & 28 & 10 & 25 & 35 & 14 & 51 & 75 \\
\hline 16 & 20 & 8 & 40 & 48 & 10 & 42 & 75 \\
\hline 17 & 20 & 5 & 33 & 38 & 28 & 34 & 72 \\
\hline 18 & 7 & 0 & 20 & 20 & 20 & 60 & 75 \\
\hline 19 & 10 & 0 & 30 & 30 & 30 & 40 & 72 \\
\hline 20 & 12 & 0 & 33 & 33 & 30 & 37 & 76 \\
\hline 21 & 8 & 0 & 33 & 33 & 43 & 24 & 75 \\
\hline 22 & 52 & 18 & 36 & 54 & 6 & 40 & 69 \\
\hline 23 & 30 & 12 & 30 & 42 & 29 & 29 & 70 \\
\hline 24 & 13 & 16 & 33 & 49 & 12 & 39 & 74 \\
\hline 25 & 32 & 20 & 39 & 59 & 18 & 23 & 70 \\
\hline 26 & 28 & 20 & 38 & 58 & 12 & 30 & 75 \\
\hline 27 & 20 & 5 & 40 & 45 & 30 & 25 & 71 \\
\hline 28 & 49 & 0 & 20 & 20 & 20 & 60 & 75 \\
\hline 29 & 17 & 18 & 34 & 52 & 8 & 40 & 70 \\
\hline 30 & 12 & 0 & 45 & 45 & 30 & 25 & 74 \\
\hline 31 & 2 & 0 & 30 & 30 & 40 & 30 & 75 \\
\hline 32 & 28 & 0 & 30 & 30 & 10 & 60 & 74 \\
\hline 33 & 24 & 5 & 30 & 35 & 25 & 40 & 75 \\
\hline 34 & 60 & 10 & 30 & 40 & 16 & 44 & 70 \\
\hline 35 & $<0$, I & 0 & 0 & 0 & 0 & 100 & 90 \\
\hline 36 & 20 & 5 & 37 & 42 & 25 & 33 & 70 \\
\hline 37 & 7 & 12 & 46 & 58 & 18 & 24 & 75 \\
\hline 38 & 70 & 12 & 38 & 50 & 14 & 36 & 70 \\
\hline 39 & 8 & 7 & 26 & 33 & 22 & 45 & 72 \\
\hline 40 & 18 & 0 & 20 & 20 & 20 & 60 & 72 \\
\hline 41 & 50 & 12 & 48 & 60 & 18 & 22 & 70 \\
\hline 42 & 5 & 0 & 33 & 33 & 30 & 37 & 72 \\
\hline 43 & 26 & 18 & 38 & 56 & 18 & 26 & 70 \\
\hline 44 & 29 & 0 & 33 & 33 & 30 & 37 & 73 \\
\hline 45 & 46 & 0 & 45 & 45 & 10 & 45 & 76 \\
\hline 46 & 21 & 10 & 35 & 45 & 5 & 50 & 72 \\
\hline 47 & 22 & 10 & 48 & 58 & 12 & 30 & 72 \\
\hline 48 & 26 & 8 & 32 & 40 & 13 & 46 & 75 \\
\hline 49 & 14 & 10 & 20 & 30 & 14 & 56 & 78 \\
\hline 50 & 0,5 & 0 & 20 & 20 & 20 & 60 & 85 \\
\hline 51 & 12 & 0 & 20 & 20 & 22 & 58 & 80 \\
\hline
\end{tabular}

Valori normali di riferimento: Conc/ml $>20 \times 10^{6}$; Motilità rettilinea $A>25 \%$; Motilità $A+B>50 \%$; Morfologia atipica $<70 \%$ 
so di identificare 2 campioni che altrimenti sarebbero risultati negativi. Si sottolinea quindi la necessità, nella diagnostica di HPV, di analizzare sempre le regioni E6/E7 e di non fermarsi all'analisi della sola regione L1 per non perdere quei campioni positivi per cui, durante l'integrazione del genoma virale nel DNA della cellula ospite, è avvenuta la perdita della regione L1.

È importante sottolineare che gli spermatozoi erano tutti infettati da genotipi HPV ad alto rischio con una prevalenza di HPV 16 e HPV 31; questo dato non sorprende perché è in accordo con i dati epidemiologici, non pubblicati, del Laboratorio di Virologia del Policlinico di Modena relativi alla circolazione di tali genotipi virali nella popolazione femminile del comprensorio modenese. Non sorprende il ritrovamento di genotipi HPV a basso rischio quali HPV 44 e HPV 81, in associazione rispettivamente con HPV 52 e HPV 31 ad alto rischio in quanto le coinfezioni sono ampiamente documentate.

La positività in 2 campioni per le sole regioni E6/E7 dei genotipi ad alto rischio HPV 16 e HPV 31 indica la presenza di infezione persistente con integrazione del DNA virale nella cellula ospite. È possibile che HPV possa essersi integrato nel DNA dello spermatozoo e possa provocare l'espressione delle proteine oncogene E6 ed E7 di HPV che a loro volta possono interagire con le proteine cellulari e compromettere molte importanti funzioni biologiche degli spermatozoi.

In merito alla presenza di HPV negli spermatozoi, nel presente studio, tale dato non sembra influenzare significativamente i parametri seminali analizzati quali la motilità, la concentrazione e la morfologia degli spermatozoi $(p>0.05)$. Tuttavia l'analisi della varianza ha permesso di rilevare una tendenza alla significatività tra HPV e oligozoospermia.

In conclusione da questo studio, che si deve ritenere preliminare data l'esiguità del numero dei campioni, emerge che il Papillomavirus è presente negli spermatozoi e può essere integrato nel DNA dei pazienti infertili considerati. Nonostante la presenza di tale virus non sembri incidere significativamente sui parametri seminali nemaspermici, una tendenza alla significatività è comunque emersa tra HPV e oligozoospermia.

Tabella 2. Distribuzione percentuale dei parametri nemaspermici dei 5 I campioni

\begin{tabular}{ccccc}
\hline Campioni & Concentrazione/ml (\%) & $\begin{array}{c}\% \text { Motilità } \\
\text { rettilinea }\end{array}$ & $\begin{array}{c}\text { \% Motilità } \\
\text { rettilinea e ondulatoria }\end{array}$ & \% Morfologia \\
\hline Normali & 29 & 0 & 10 & 1 \\
& $(57 \%)$ & $(0 \%)$ & $(20 \%)$ & $(2 \%)$ \\
\hline Patologici & 22 & 51 & 41 & 50 \\
& $(43 \%)$ & $(100 \%)$ & $(80 \%)$ & $(98 \%)$ \\
\hline
\end{tabular}

Tabella 3. Campioni positivi all'amplificazione dei geni LI e E6/E7 e genotipizzazione

\begin{tabular}{|c|c|c|c|}
\hline \multirow{3}{*}{$\begin{array}{c}\text { Campioni } \\
\text { Positivi }\end{array}$} & \multicolumn{2}{|c|}{ PCR gene LI } & \multirow[t]{2}{*}{ PCR geni E6/E7 } \\
\hline & Linear Array ${ }^{\circledR}$ & & \\
\hline & HPV Genotyping & INNO-LiPA & RFLP \\
\hline 1 & HPV 16 & & \\
\hline 2 & Non tipizzabile & HPV 52,44 & \\
\hline 3 & Non tipizzabile & HPV 16 & \\
\hline 4 & HPV 73 & & \\
\hline 5 & HPV 3I & & \\
\hline 6 & HPV 3I,8I & & \\
\hline 7 & Non tipizzabile & Non tipizzabile & \\
\hline 8 & & & HPV 3I \\
\hline 9 & & & HPV 3I \\
\hline
\end{tabular}

Tabella 4. Risultati relativi all'associazione tra positività virale e parametri seminali

\begin{tabular}{ccccccccc}
\hline & \multicolumn{3}{c}{ Conc./ml (\%) } & \multicolumn{2}{c}{ Motilità rettilinea (\%) } & \multicolumn{2}{c}{$\begin{array}{c}\text { Motilità rettilinea } \\
\text { e ondulatoria (\%) }\end{array}$} & \multicolumn{2}{c}{ Morfologia (\%) } \\
\hline Campioni & Patologici & Normali & Patologici & Normali & Patologici & Normali & Patologici & Normali \\
\hline HPV Pos & $6(30 \%)$ & $3(10 \%)$ & $9(18 \%)$ & $0(0 \%)$ & $6(15 \%)$ & $3(30 \%)$ & $9(19 \%)$ & $0(0 \%)$ \\
\hline HPV Neg & $14(70 \%)$ & $26(90 \%)$ & $40(82 \%)$ & $0(0 \%)$ & $33(85 \%)$ & $7(70 \%)$ & $39(81 \%)$ & $1(100 \%)$ \\
\hline Totale & $20(41 \%)$ & $29(59 \%)$ & $49(100 \%)$ & $0(0 \%)$ & $39(80 \%)$ & $10(20 \%)$ & $48(98 \% 9$ & $I(2 \%)$ \\
\hline p $>0.05$ & & & & & & &
\end{tabular}




\section{BIBLIOGRAFIA}

1. Bauer MH, Ting Y, Greer EG, et al. Genital Human Papillomavirus infection in female university students as determined by a PCR-based method. JAMA 1991; 265: 23-30

2. Foresta C, Pizzol D, Moretti A, Barzon L, Palù G, Garolla A. Clinical and prognostic significance of human papilloma virus DNA in the sperm or exfoliated cells of infertile patients and subjects with risk factors. Fertil Steril 2010; 94: 1723-7

3. Jacobs MV, de Roda Husman AM, van den Brule AJ, Snijders PJ, Meijer CJ, Walboomers JM. Group specific differentiation between High- and Low-Risk Human Papillomavirus Genotypes by general Primermediated PCR and two cocktails of oligonucleotide probes. J Clin Microbiology 1995; 33: 901-5.

4. Lai YM, Lee JF, Huang HY, Soong YK, Yang FP, Pao CC. The effect of human papillomavirus infection on sperm cell motility. Fertil Steril 1997; 67: 1152-5

5. Noda T, Sassagawa T, Dong Y, Fuse H, Namiki M, Inoue M. Detection of human papillomavirus (HPV) DNA in archival specimen of benign prostatic hyperplasia and prostatic cancer using highly sensitive nested PCR method. Urol Res 1998; 26: 165-9.

6. Nyitray A. Anal cancer and human papillomaviruses in heterosexual men. Curr Oncol 2008; 15: 204-5.

7. Oh JK, Franceschi S, Kim BK et al. Prevalence of human papillomavirus and Chlamydia trachomatis infection among women attending cervical cancer screening in the Republic of Korea. Eur 1 Cancer Prev 2009; 18: 56-61.

8. Ostrow RS, Zachow KR, Niimura M, et al. Detection of papillomavirus DNA in human semen. Science 1986; 31: 731-3

9. Pao CC, Lai YM, Yang FP et al. Preferential retention of the E6 and E7 regions of the human papilloma virus type 18 genome by human sperm cells. Fertil Steril 1996; 66: 630-3.

10. Rintala MA, Grénman SE, Pöllänen PP, Suominen JJ, Syrjänen SM. Detection of high-risk HPV DNA in semen and its association with the quality of semen. Int J STD AIDS 2004; 15: 740-3.

11. World Healt Organization. Laboratory manual for the examination of human semen and sperm cervical mucus interaction. Cambridge: Cambridge University Press 1999.

12. Zazzi M, Catucci M, De Milito A,et al. Zidovudina resistence mutations and human immunodefiency virus type 1 DNA burden: longitudinal evaluation of six patients under treatment. Infection 1996; 24: 419-25. 